\title{
Estudio preliminar de la arácnofauna en la ribera del río Chinguiñoso, Huejutla de Reyes, Hidalgo, México
}

Preliminary study of arachnids in the river bank Chinguiñoso, Huejutla de Reyes, Hidalgo, Mexico

Cipriano-Anastasio Juan ${ }^{1 凶}$, Cruz-Salazar Fausta Laura ${ }^{1}$, López-Mancilla Alejandra ${ }^{1}$, HernándezHernández, Jesús ${ }^{1}$, Lara-Hernández, José Luis ${ }^{1}$ y Cruz-Monterrubio, Andrea Leonor ${ }^{1}$.

${ }^{1}$ Instituto Tecnológico de Huejutla. Carretera Huejutla-Chalahuiyapa Km 5.5, CP. 43000, Huejutla de Reyes, Hidalgo, México.

${ }^{凶}$ Autor para correspondencia: bio.jca@gmail.com

Recibido: 11/09/2019

Aceptado: 22/11/2019

\section{RESUMEN}

Las arañas comprenden un grupo de fauna muy diverso y ampliamente distribuido en todos los ecosistemas terrestres invadiendo incluso algunos ambientes dulceacuícolas. Se realizó un estudio sobre la arácnofauna en la ribera del río Chinguiñoso, Huejutla de Reyes, Hidalgo; México. Durante los meses de septiembre a noviembre del 2017. Se utilizó el método de colecta directa, trampas de caída y golpeo de ramas y arbustos, en un trayecto de dos kilómetros, distribuidos en cinco sitios cada uno con 200 metros de longitud, obteniendo 240 horas de esfuerzo de muestreo en jornadas diurnas. Se obtuvieron un total 190 individuos, distribuidos en 13 especies y siete familias (Araneidae, Oxyopidae, Pisauridae, Salticidae, Sytodidae, Sparissidae y Tetragnathidae). Se tomó como medida de la diversidad verdadera (especies efectivas) la exponencial del índice de equidad de Shannon (H'); a su vez dominancia de Simpson (D’), como un indicador para medir la diversidad. El mes de septiembre fue el más abundante, rico y diverso. Por otra parte, el análisis de similitud de Jaccard arroja un grupo que presenta un $80 \%$ de similitud y otro en donde presenta especies que no se encontraron en los demás sitios. La riqueza y diversidad de arácnidos podría estar aunada a la riqueza de vegetación, debido a que aún existen zonas con gran altura y cobertura arbórea.

Palabras clave: Riqueza, diversidad verdadera, arácnidos, equidad y dominancia.

\begin{abstract}
Spiders comprise a group of very diverse and widely distributed fauna in all terrestrial ecosystems invading even some freshwater environments. A study was carried out on the arachnofauna on the banks of the Chinguiñoso River, Huejutla de Reyes, Hidalgo; Mexico. During the months of September to November of 2017. The method of direct collection, fall traps and beating of branches and bushes was used, in a two-kilometer journey, distributed in five sites each with 200 meters in length, obtaining 240
\end{abstract}


hours of sampling effort in daytime sessions. A total of 190 individuals were obtained, distributed in 13 species and seven families (Araneidae, Oxyopidae, Pisauridae, Salticidae, Sytodidae, Sparissidae and Tetragnathidae). The exponential of Shannon's equity index ( $\mathrm{H}^{\prime}$ ) was taken as a measure of true diversity (effective species); Simpson's dominance (D '), as an indicator to measure diversity. The month of September was the most abundant, rich and diverse. On the other hand, Jaccard's similarity analysis shows a group that presents $80 \%$ similarity and another group that presents species that were not found in the other sites. The richness and diversity of arachnids could be coupled with the richness of vegetation, because there are still areas with high altitude and tree cover.

Keywords: Richness, true diversity, arachnids, equity and dominance.

\section{INTRODUCCIÓN}

Las aguas dulces constituyen un hábitat donde viven y se desarrollan gran variedad de seres vivos, los cuales dependen del agua para su subsistencia. Las arañas, después de los insectos y crustáceos forman uno de los grupos de invertebrados más diversos del mundo, aunque es probable que el número de especies llegue a 50,000 (Turnbull, 1973). Por su amplia capacidad de adaptarse al medio, su gran abundancia, facilidad para dispersarse y el variado uso de la seda, las arañas han colonizado prácticamente todos los hábitats terrestres; se pueden encontrar desde el ecuador hasta los polos y desde los desiertos hasta los cuerpos de agua (Chan et al. 2009). Debido a sus hábitos, pueden ser perseguidoras o acechadoras. Con respecto a su hábitat unas prefieren vivir en las flores y hojas o bajo las rocas y cortezas de los árboles viejos, muchas tienen una preferencia marcada por algún tipo de vegetación o por plantas específicas. Los ambientes ribereños pueden ser un buen indicador de las poblaciones de arácnidos, debido a que cuentan con la vegetación apropiada tal es el caso de árboles y arbustos. Algunas forman grupos sociales
(Aguilera y Casanueva 2005). El conocimiento de la dinámica poblacional indica que las arañas actúan como agentes estabilizadores de poblaciones de artrópodos (Crespo y Ramírez, 2011) y por lo tanto de los ecosistemas en los que habitan. Su acción puede ser eficaz en la prevención del desarrollo de poblaciones elevadas de plagas de insectos reduciendo el uso de insecticidas y por lo tanto el costo de los cultivos (Jiménez, 1996). El presente estudio tiene como objetivo determinar la diversidad de arácnidos en la ribera del río Chinguiñoso.

\section{MATERIAL Y MÉTODOS}

El río Chinguiñoso se ubica en la localidad de Huejutla de Reyes, al norte del estado de Hidalgo, México. Entre los paralelos $21^{\circ} 08^{\prime} 24^{\prime \prime}$ de latitud Norte y $98^{\circ} 25^{\prime} 10^{\prime \prime}$ de longitud Oeste, a una altitud de 137 metros sobre el nivel del mar. El Municipio registra un clima semicálido húmedo con abundantes lluvias en verano y una temperatura media anual de $31.1^{\circ} \mathrm{C}$. La precipitación pluvial es de 1,500-2000 milímetros por año. Pertenece a la provincia fisiográfica Sierra Madre Oriental y llanura Costera del Golfo Norte (Fig. 1) (INEGI, 2015). 


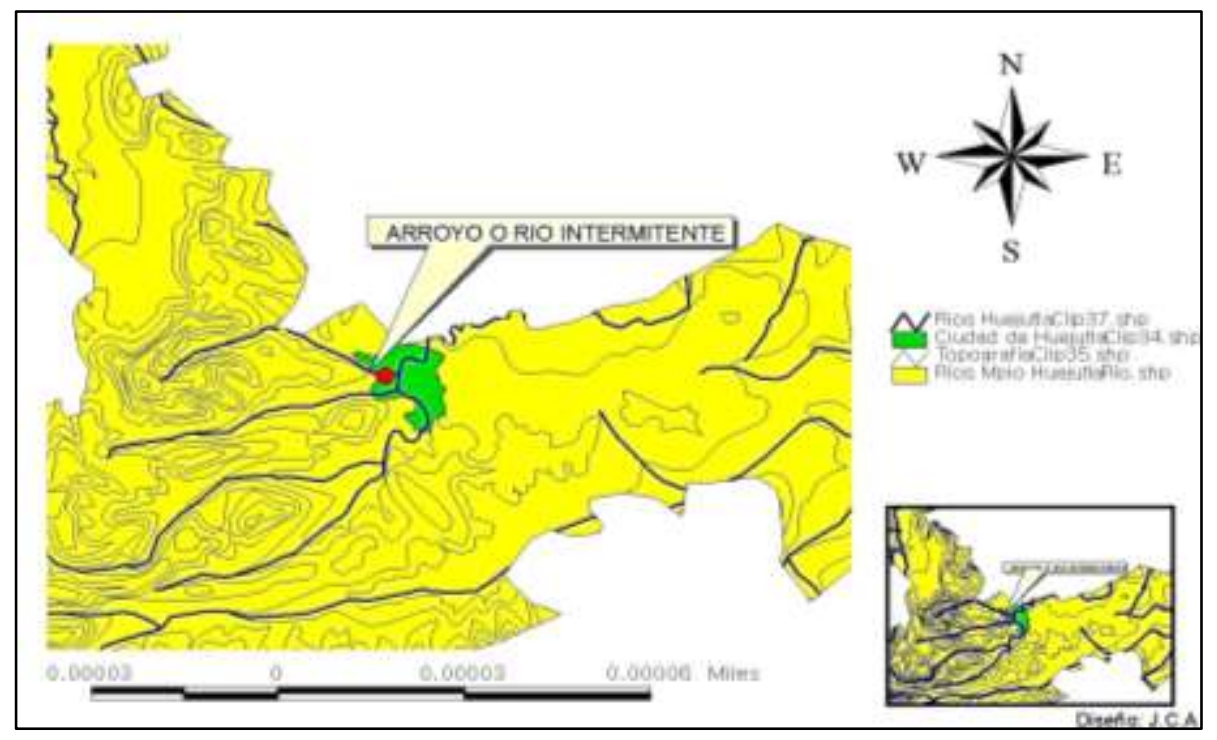

Figura 1. Localización del río Chinguiñoso.

Trabajo de campo. Durante el periodo septiembre-noviembre del 2017. Se realizaron un total de seis salidas, obteniendo un total de $240 \mathrm{~h}$ de esfuerzo de muestreo. Dentro de la ribera del río chinguinoso se marcó un transecto de $200 \mathrm{~m}$ por sitio, recorriendo un total de 1000 m. Se aplicaron técnicas de muestreo de arácnidos en la vegetación riparia (colecta directa), se realizaron golpeos de ramas, revisión de arbustos pequeños y grandes, se colocaron trampas de caída (Márquez, 2005). Los especímenes colectados fueron sacrificados dentro de un frasco con formol. Para la determinación taxonómica se utilizaron guías de Arácnidos, en la comparación de los ejemplares y el análisis de diversidad solo se utilizaron los especímenes adultos debido a que los especímenes juveniles no presentan características diagnosticas exclusivas de genitales por lo que no pueden ser determinados especie.

Análisis de datos. La riqueza de especies se basa en el número total de especies obtenido por un censo de la comunidad (Moreno, 2001). Se tomó la medida de diversidad verdadera de orden uno (q1) en la cual todas las especies son consideradas en el valor de diversidad, ponderadas proporcionalmente según su abundancia en la comunidad (Moreno et al. 2011), por medio de la obtención exponencial del índice de entropía de Shannon: ${ }^{1} \mathrm{D}=\exp \left(\mathrm{H}^{\prime}\right)$ (Jost, 2006). Para el Análisis de similitud, se empleó el coeficiente de similitud de Jaccard para determinar el grado en que dos muestras son semejantes por las especies presentes en ellas. Donde el rango de este índice va desde cero cuando no hay especies compartidas, hasta uno cuando los dos sitios comparten las mismas especies. Este índice mide diferencias en la presencia o ausencia de especies.

\section{RESULTADOS}

Durante 240 horas de muestreo se obtuvieron un total de 190 individuos, 13 especies y siete familias (Araneidae, Oxyopidae, Pisauridae, Salticidae, Sytodidae, Sparissidae y Tetragnathidae). El mes con mayor abundancia y riqueza fue noviembre, las especies más representativas fueron: Tetragnata elongada, Argiope argentata, Peucettia viridans $\mathrm{y}$ 
Phidippus audax. La diversidad verdadera nos indica que en este mismo mes se obtuvieron 5.3 especies efectivas, siendo este el más representativo (Fig. 2).

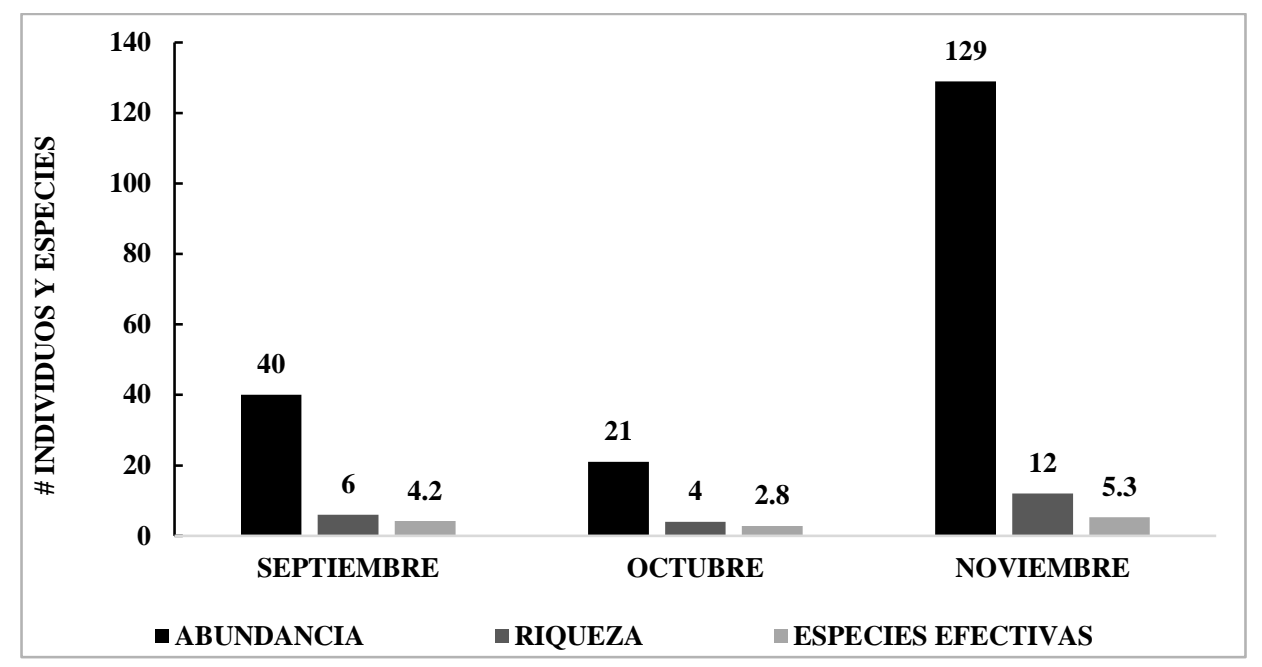

Figura 2. Abundancia, riqueza y especies efectivas mensuales.

Abundancia, riqueza y diversidad verdadera por sitio

El sitio tres obtuvo mayor abundancia, riqueza y especies efectivas. Las especies más representativas fueron: T. elongada, Peucettia viridans Phidippus audax (Fig. 3).

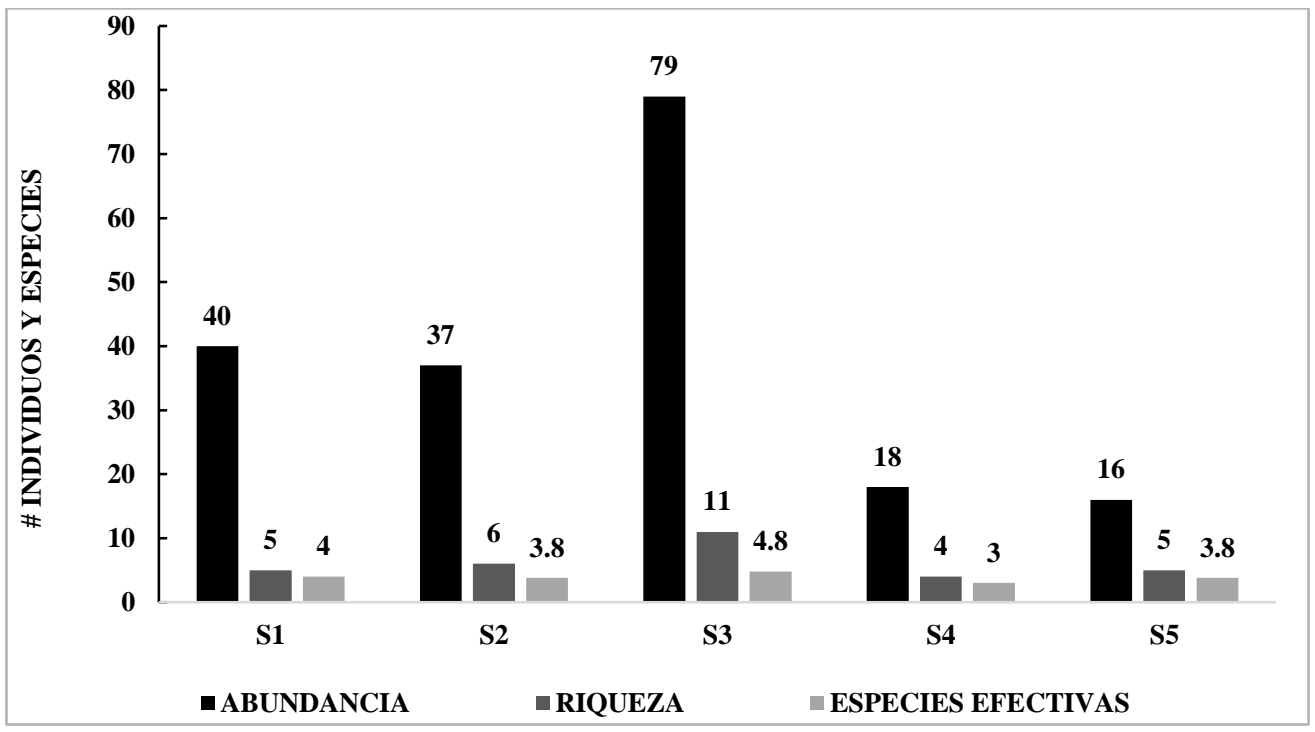

Figura 3. Abundancia, riqueza y especies efectivas por sitio.

En el mes de septiembre la equidad fue mayor, en octubre descendió y en noviembre nuevamente ascendió. La dominancia en el mes de septiembre fue baja, en octubre ascendió y en noviembre descendió nuevamente (Fig. 6). Esto indica un aumento en la equidad para este último mes. 
Cipriano-Anastasio et al., 2019

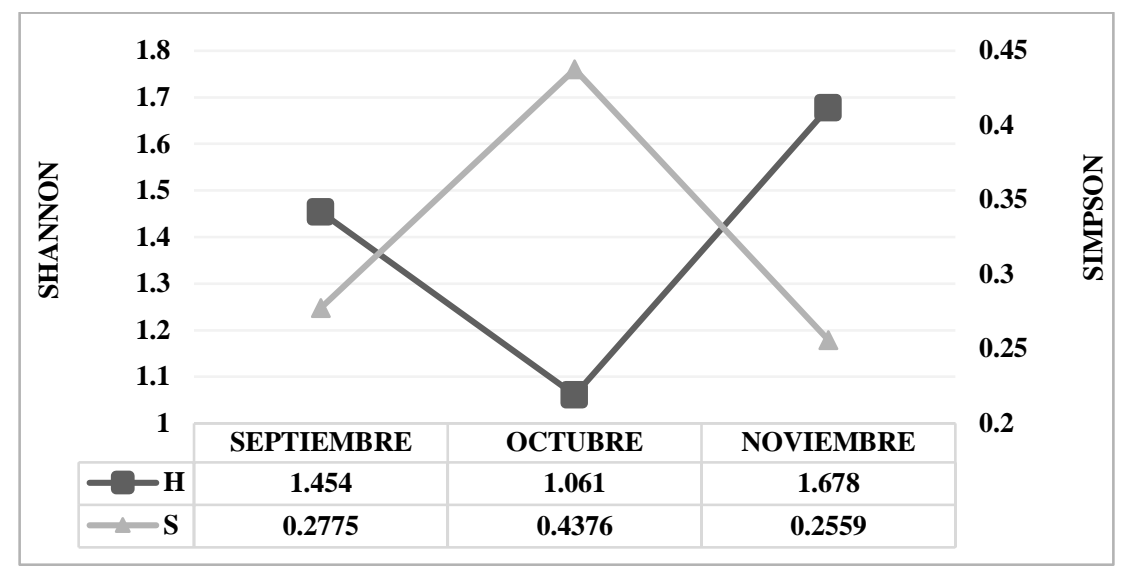

Figura 4. Índice de equidad de Shannon y dominancia de Simpson mensual y por sitios.

El sitio uno presenta una mayor equidad, en el sitio dos descendió. El sitio tres ascendió dominancia el sitio uno, tres y cinco, presentaron nuevamente, siendo el más diverso. En la menor valor que el sitio dos y cuatro (Fig. 5).

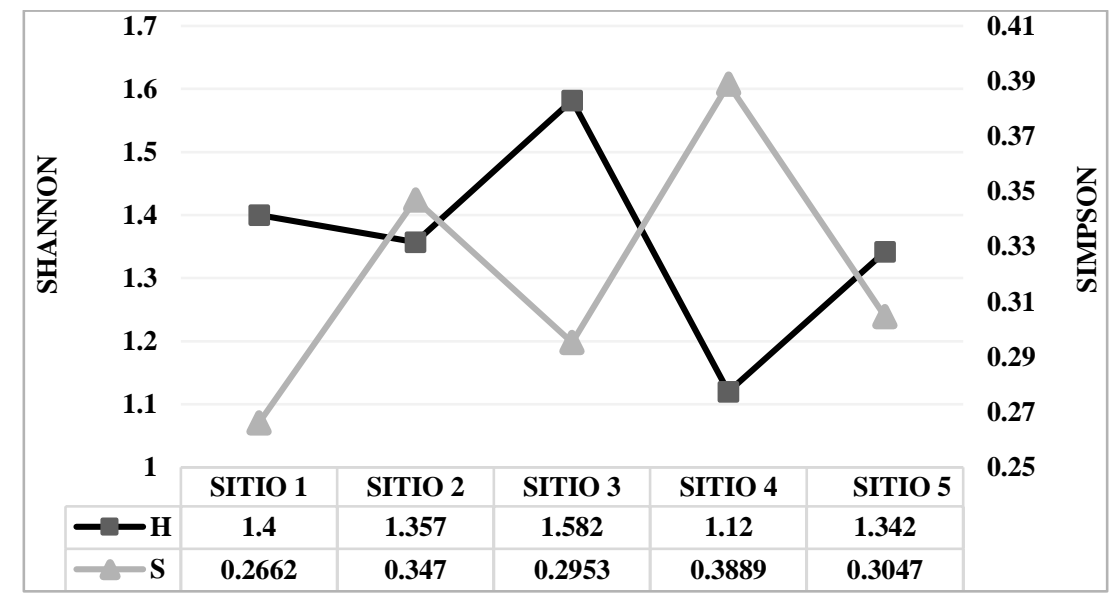

Figura 5. Índice de equidad de Shannon y dominancia de Simpson por sitios.

\section{Curva de acumulación de especies}

De acuerdo a los estimadores Jack 1 y Jack 2 la muestra uno coincide con el número de especies estimadas. En nuestra muestra dos se obtuvieron seis especies y de acuerdo con los estimadores debieron obtenerse siete. En la muestra tres, se estiman nueve y diez. En la muestra cuatro, nueve especies. Para el quinto y sexto muestreo se estimaron entre 17 y 22 especies (Fig. 6). 
Cipriano-Anastasio et al., 2019

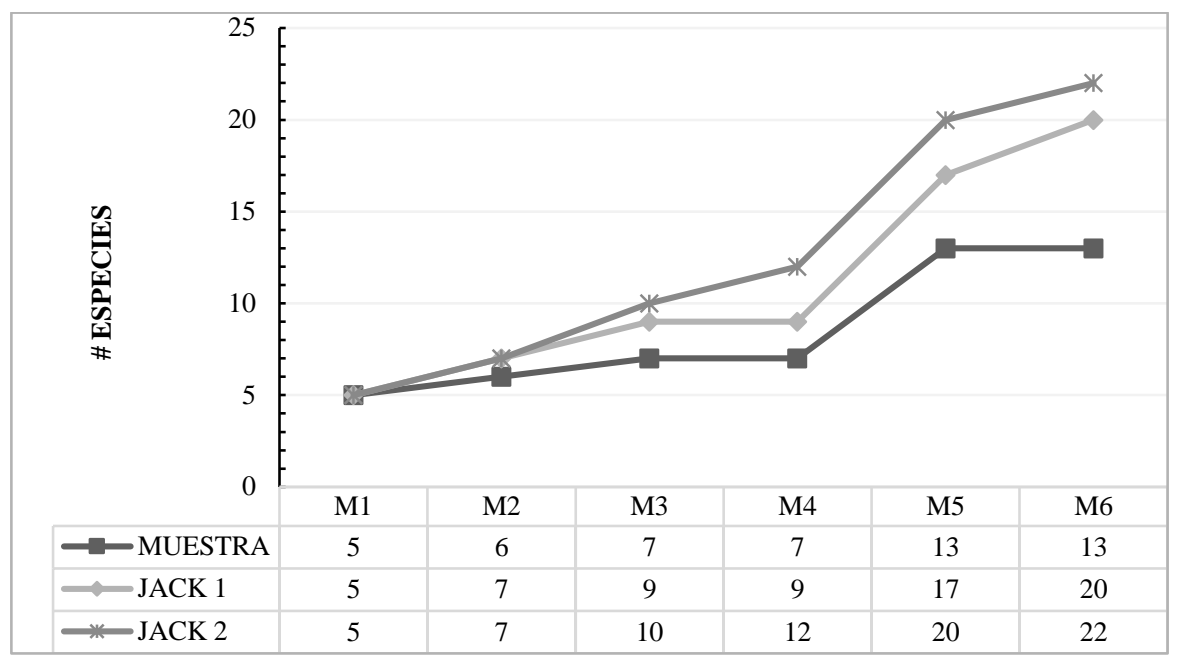

Figura 6. Curva de acumulación de especies.

Análisis de similitud. La similitud en el grupo cuatro y uno es de 0.80 (80\%), teniendo especies en común: C. hesperus, P. audax y T. elongada. El grupo cinco y dos presenta una similitud de $0.56(56 \%)$. El sitio tres presenta especies que no se encuentran en los demás sitios: E. ravilla, L. jemineus, $S$. fusca y $T$. peregrinus (Fig. 7).

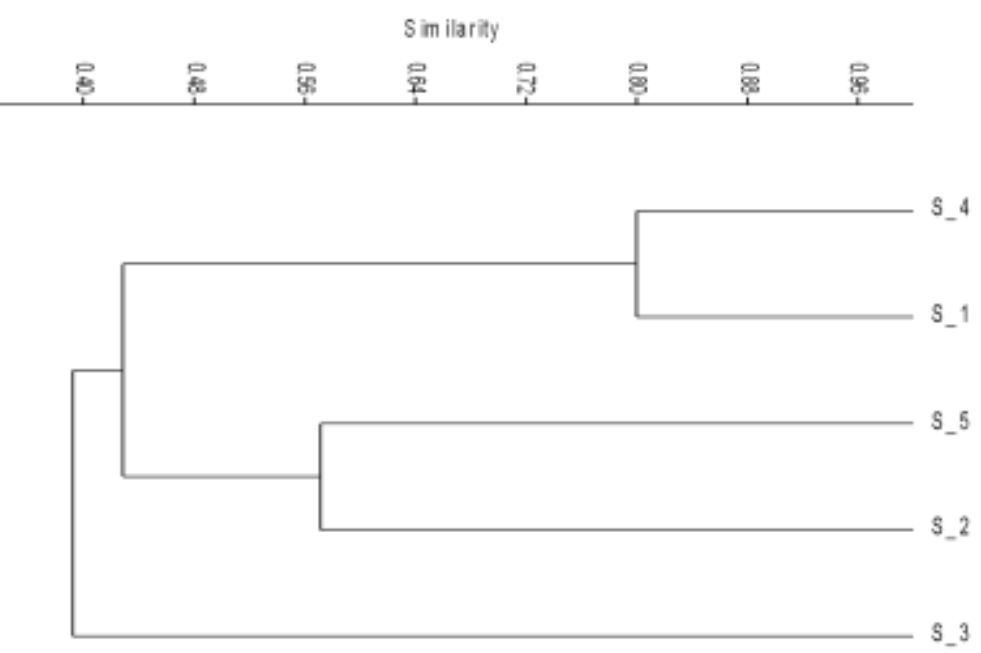

Figura 7. Análisis de similitud de Jaccard en la ribera del río Chinguiñoso.

\section{DISCUSIÓN}

Este estudio estableció los primeros datos de diversidad de arácnidos presentes en la ribera del río Chinguiñoso, Huejutla de Reyes, Hidalgo. Se hace mención que no existen trabajos relacionados a este grupo en la zona, siendo este el primero. Por lo que discutimos nuestro estudio 
con investigaciones realizadas en otros hábitats similares.

Se registran siete familias y 13 especies de arácnidos en la zona. Los resultados muestran que Peucetia viridans y Phidippus audax fueron las especies más representativas de la zona y contribuyeron a la diversidad. Por otra parte, hubo especies que presentaron una mayor dominancia como T. elongada, debido a que son de amplia distribución y sus hábitats en ambientes húmedos responden a sus adaptaciones para obtener alimento, así como también a su historia natural (Döbel et al. 1990). Por otro lado, los géneros Salticidae reportados en este trabajo son de alta distribución y no se restringen a otros sitios, esto explica en base a la diversidad de microhábitats que ofrece la ribera del río Chinguiñoso, además de otros aspectos ecológicos que determinan la selección de hábitats de las arañas. Se observa, además, que la comunidad de arañas en el área de estudio está representada por familias consideradas de amplia distribución como los son Araneidae, Salticidae, Tetragnathidae (Dippenaar et al. 1999), información que coincide con los resultados obtenidos en estudios de diversidad realizados en distintas comunidades de arañas (Cepeda y Flórez, 2007). Estas familias son unas de las más diversas a nivel mundial, y se caracterizan por presentar especies con capacidad de explotar los diversos recursos disponibles.

El monitoreo en ambientes húmedos muestra la utilidad de las arañas para futuros trabajos (Reyes-Maldonado en imprenta). En este trabajo la mayor riqueza y diversidad fue encontrada en zonas alejadas a la mancha urbana. Esto es un reflejo de la disponibilidad alimenticia o cambios en la vegetación. Un estudio realizado por Chan et al. (2009) descubrieron que las arañas ribereñas aumentaron significativamente en lugares donde disponían de sustrato adicional es decir mayor vegetación que en otros sitios muestreados. Existen zonas ribereñas alteradas debido a la mancha urbana, esto provoca cambios en comunidades de insectos acuáticos (Crespo y Ramírez, 2011).

Una medida de amplio uso para evaluar la diversidad es la riqueza de especies (Magurran, 2004). Trabajar con la riqueza de especies es más adecuado que la sola abundancia para describir la comunidad o la diversidad regional o local (Gotelli y Colwell, 2001). El esfuerzo y la cobertura del muestreo respecto al hábitat y al organismo estudiado, así como la heterogeneidad ambiental son algunos factores que influyen en la medición y estimación de la riqueza (Palmer, 1990). El uso de los números efectivos permitió una mejor interpretación de la diversidad de las comunidades y las comparaciones entre sitios y meses (Moreno, 2011; Jost 2007). El índice de entropía de Shannon no tiene una explicación biológica clara, es por eso que se utilizó la medida de diversidad verdadera.

Lo anterior descrito, nos indica que la riqueza y diversidad de especies de arácnidos es mayor en donde existe gran variedad de árboles presentes y estas a su vez corresponden fuertemente a la urbanización. El estudio de similitud no pudo ser comparado con otros trabajos, debido a que la mayoría se enfoca en áreas mayormente terrestres. Es importante estudiar los ecosistemas ribereños, debido a que albergan una gran diversidad de arácnidos que utilizan estos ambientes para realizar sus necesidades biológicas. 


\section{CONCLUSIONES Y RECOMENDACIONES}

Se obtuvieron un total de 190 individuos, 13 especies y siete familias (Araneidae, Oxyopidae, Pisauridae, Salticidae, Sytodidae, Sparissidae y Tetragnathidae). El mes con mayor abundancia y riqueza fue noviembre. El sitio más rico y diverso fue el tres, nuestra suposición es que el sitio cuenta con una gran cobertura vegetal, brindando un hábitat adecuado para la arácnofauna. La curva de acumulación de especies nos indica que faltan especies por registrar, en los meses de octubre y noviembre se vieron afectados los muestreos por la creciente del río. La información obtenida en la realización de este estudio aporta conocimiento sobre las diversas especies de arácnidos que se encuentran en la ribera del río Chinguinoso Huejutla de Reyes Hidalgo y la importancia ecológica de ellas como controladoras de plagas con ayuda de sus telarañas que son redes de captura para diversos insectos como Aedes aegypty el cual es un vector de enfermedades como el dengue, sika y chincongunya que afectan a la población durante el estadio maduro. Lo más recomendable para el aprovechamiento de estos arácnidos es dejar que sigan su proceso biológico natural, debido a los beneficios ecológicos que estos aportan.

\section{LITERATURA CITADA}

Aguilera, M. A. y Casanueva, M. E. 2005. Arañas chilenas: estado actual del conocimiento y clave para las familias Araneomorphae. Gayana 69 (2): 201-224. https://doi.org/10.4067/S0717-6538200500 0200001

Cepeda V. J. y Flores D., E. 2007. Arañas tejedoras: uso de diferentes microhábitats en un bosque andino de Colombia. Revista Ibérica de Aracnología 14 (31): 39-48.
Chan, E. K.; Zhang, Z.; Dudgeon, D. 2009. Substrate Availability May Be More Important than Aquatic Insect Abundance in the Distribution of Riparian Orb-web Spiders in the Tropics. Biotrópica 41 (2): 196-201. https://doi.org/10.1111/j.1744-74 Dippenaar-Schoeman, A.S., Van den Berg, A.M. \& Van den Berg, A. 1999. Spiders in South African cotton fields: species diversity and abundance (Arachnida: Araneae). African Plant Protection 5: 93-103

Döbel, H. G.; Denno, R. F.; Coddington, J. A. 1990. Spider (Araneae) community structure in an intertidal salt marsh: Effects of vegetation structure and tidal flooding. Environmental Entomology 5 (19): 13561370. https://doi.org/10.1093/ee/19.5.1356

Foelix, F. R. 1996. Biology of spiders. Editorial Oxford, New York.

Gotelli, N. J. and Colwell, R. K. 2001. Quantifying biodiversity: procedures and pitfalls in the measurement and comparison of species richness. Ecology Letters 4: 379-391. https://doi.org/10.1046

INEGI. 2015. Anuario estadístico del estado de Hidalgo. Instituto Nacional de Estadística y Geografía, México.

Crespo R. y Ramírez, A. 2011. Effects of urbanization on stream physicochemistry and macroinvertebrate assemblages in a tropical urban watershed in Puerto Rico. Journal of the North American Benthological Society 30 (3): 739-750. https://doi.org/10.1899/10-081.1

Jiménez, M. L. 1996. Araneae. Págs. 83-101 en: Llorente,-Bousquets J. E.; García-Aldrete, A. N.; González-Soriano, E. (Eds). Biodiversidad, taxonomía y biogeografía de ártropodos de México: Hacia una sintesis de su conocimiento. Instituto de Biología, UNAM, México, D.F. 


\section{Cipriano-Anastasio et al., 2019}

Jost, L. 2007. Partitioning diversity into independent alpha and beta components. Ecology $88 \quad$ (10): 2427-2439. https://doi.org/10.1890/06-1736.1

Levi, H. W.; Coddington, J. 1991. Systematics and evolution of spiders (Araneae). Annual Review of Ecology and Systematics 22:

565-92. https://doi.org/10.1146/annurev.es.22.110 191.003025

Márquez, L. J. 2005. Técnicas de colecta y preservación de insectos. Boletín Sociedad Entomológica Aragonesa 1 (37): 385 408.

Moreno, C. E. 2001. Métodos para medir la biodiversidad. M\&T-Manuales y Tesis SEA, vol. 1. Zaragoza, 84 p.

Moreno, C. E.; Barragán, F.; Pineda, E.; Pavón, N. 2011. Reanálisis de la diversidad alfa: alternativas para interpretar y comparar información sobre comunidades ecológicas. Revista Mexicana de Biodiversidad 82 (4): 1249-1261.

https://doi.org/10.22201/ib.20078706e.201 1.4.745

Palmer, E. W. 1990. The estimation of species richness by extrapolation. Ecology 71 (3), 1195-1198.

https://doi.org/10.2307/1937387

Pérez, C. M. y Cruz, P. A. 2005. Diversidad de Teridiidos (Araneae: Theridiidae) en cuatro asociaciones florísticas, en el ejido "Las Delicias", Teapa, Sureste de México. Universidad y Ciencia 21 (41): 41-44.

Reyes, M. R.; Sánchez, R. J.; Ramírez, A.; Kelly, S. P. En imprenta. Riparian spider communities as indicators of stream ecosystem condition in the Río Piedras watershed of Puerto Rico. Actual Biology.

Turnbull, A. L. 1973. Ecology of the true spiders (Araneomorphae). Ann.Rev.Ent. 18- 305348.

https://doi.org/10.1146/annurev.en.180101 73.001513

Copyright (c) 2019 Juan Cipriano-Anastasio, Fausta Laura Cruz-Salazar, Alejandra López-Mancilla, Jesús Hernández-Hernández, José Luis Lara-Hernández, Andrea L eonor Cruz-Monterrubio

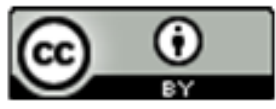

Este texto está protegido por una licencia licencia CreativeCommons 4.0 .

Usted es libre para Compartir — copiar y redistribuir el material en cualquier medio o formato- y Adaptar el documento — remezclar, transformar y crear a partir del material- para cualquier propósito, incluso para fines com erciales, siempre que cumpla la condición de:

Atribución: Usted debe dar crédito a la obra original de manera adecuada, proporcionar un enlace a la licencia, e indicar si se han realizado cambios. Puede hacerlo en cualquier forma razonable, pero no de forma tal que sugiera que tiene el apoyo del licenciante o lo recibe por el uso que hace de la obra.

Resumendelicencia - Textocompletodelalicencia 